\title{
Associação entre força e aptidão cardiorrespiratória é mais forte em septuagenários
}

\section{Correlation between muscular strength and cardiorespiratory fitness is strongest in septuagerians}

Daniel Gomes da Silva Machado 1,2, Hassan Mohamed Elsangedy², Pedro Moraes Dutra Agrícola 2,3, Luiz Fernando de Farias Junior ${ }^{2}$, Luiz Inácio do Nascimento Neto ${ }^{1,2}$, André Igor Fonteles ${ }^{2,4}$, Cheng Hsin Nery Chao², Eduardo Caldas Costa², Edilson Serpeloni Cyrino2, Paulo de Tarso Veras Farinatti, ${ }^{5,6}$, Alexandre Hideki Okano ${ }^{1,2}$

\section{Resumo}

O propósito deste estudo foi analisar a associação entre a força muscular e aptidão cardiorrespiratória em diferentes grupos etários. Duzentos e trinta e seis participantes (75 homens e 161 mulheres) de 40 a 84 anos foram divididos em quatro grupos etários: 40-49, 50-59, 60-69, $\geq 70$ anos. A força muscular foi avaliada pelo teste de força de preensão manual (FPM), enquanto a aptidão cardiorrespiratória foi estimada pela distância percorrida no teste de caminhada de 6-min. Uma correlação positiva e significante foi encontrada entre a FPM e distância percorrida no teste de caminhada de 6-min somente no grupo $\geq 70$ anos $(\mathrm{r}=0,51 ; p=0,01)$. Quando combinados em um modelo de regressão múltipla, a $\operatorname{FPM}(p=0,01)$ e o gênero $(p=0,36)$ explicaram $47,4 \%$ da variância na distância percorrida no teste de caminhada de 6-min no grupo $\geq 70$ anos. Os resultados sugerem que a força muscular parece ser um importante determinante da aptidão cardiorrespiratória em indivíduos $\geq 70$ anos, independente do gênero.

\section{Palavras-chave}

Envelhecimento; Idosos; Atividade física.

\begin{abstract}
The purpose of this study was to analyze the association between muscular strength and cardiorespiratory fitness in different age groups. Two bundred thirty-six subjects ( 75 men and 161 women) from 40 to 84 years old were divided in four age groups: 40-49, 5059, 60-69, and $\geq 70$ years old. Muscular strength was assessed by handgrip (HG), while cardiorespiratory fitness was estimated by the distance walked in the 6-min walk test. A positive significant correlation was found between $H G$ and distance walked in the 6-min walk test only in the $\geq 70$ years group $(r=0.51 ; p=0.01)$. A multiple regression analysis combining $H G(p=0.01)$ and gender $(p=0.36)$ explained $47.4 \%$ of the variance in the distance walked in the 6-min walk test in the $\geq 70$ group. This result suggest that muscular strength seems to be an important determinant of cardiorespiratory fitness in individuals 70 years and older, independent of gender.
\end{abstract}

\section{Keywords}

Aging; Elderly; Physical Activity.

\section{Introdução}

O processo de envelhecimento é caracterizado por inúmeras mudanças, incluindo o decréscimo de desempenho físico ${ }^{1-3}$. Nesse sentido, tanto a força muscular quanto a aptidão cardiorrespiratória (ACR) tendem a sofrer reduções na ordem de aproximadamente $10 \%$

1 Universidade Estadual de Londrina. Centro de Educação Física e Esporte (CEFE). Londrina, Paraná, Brasil.

2 Universidade Federal do Rio Grande do Norte. Departamento de Educação Física. Natal, Rio Grande do Norte, Brasil.

3 Faculdade Maurício de Nassau, Natal, Rio Grande do Norte, Brasil. 4 Universidade de Fortaleza (Unifor), Fortaleza, Cerá, Brasil.

5 Universidade do Estado do Rio de Janeiro. Instituto de Educação Física e Esporte. Rio de Janeiro, Rio de Janeiro, Brasil

6 Universidade Salgado de Oliveira, Programa de Pós-Graduação em Ciências da Atividade Física, Niterói, Rio de Janeiro, Brasil. por década, independente do gênero ${ }^{1,4}$. Assim, idosos com maior ACR e força muscular tendem a apresentar menos limitações físicas ${ }^{5}$ e menor dependência funcional ${ }^{6}$, além de serem menos susceptíveis ao desenvolvimento de algumas doenças, tais como cardiopatias e alguns tipos de câncer ${ }^{7,8}$. Por outro lado, valores reduzidos de força muscular e ACR têm sido sugeridos como importantes preditores de morte por todas as causas ${ }^{9,10}$.

Considerando que a redução da força musculare ACR ocorrem de maneira relativamente similar com o avanço da idade em adultos, é plausível acreditar que exista uma associação entre essas variáveis. Apesar de alguns estudos terem relatado possíveis associações entre força ou massa magra e ACR, tais investigações se limitaram a analisar 
grupos etários e/ou gêneros específicos ou, ainda, analisaram amostras com amplo intervalo de idades como um todo ${ }^{1,2,11-16}$. Portanto, tais informações podem não ser representativas do comportamento dessa associação ao longo do tempo, de modo que tais estudos não permitem, por exemplo, identificar se em um grupo etário a ACR depende mais da força muscular do que em outros, informação que pode auxiliar sobremaneira na tomada de decisão para a prescrição de exercícios físicos em diferentes populações.

Com base nas informações apresentadas, o objetivo do presente estudo foi avaliar a associação entre a força muscular e ACR em diferentes grupos etários. As nossas hipóteses são de que existe correlação diretamente proporcional entre força e ACR e que a magnitude da correlação tende a ser maior com o avançar da idade.

\section{Métodos}

Os participantes do presente estudo foram recrutados em um programa de promoção de atividade física oferecido pela Universidade Federal do Rio Grande do Norte. Inicialmente, 274 indivíduos demonstraram interesse em participar do estudo. Para serem incluídos no estudo os participantes deveriam ter idade igual ou superior a 40 anos, serem assintomáticos e capazes de realizar os testes físicos. Trinta e oito participantes foram excluídos das análises devido à ausência de informações ou não comparecimento as sessões de avaliação. Assim, a amostra final foi composta por 236 indivíduos (161 mulheres e 75 homens) com idades de 40 a 84 anos. As características gerais dos participantes são apresentadas na Tabela 1. Todos os participantes após serem informados sobre os objetivos do estudo e procedimentos aos quais seriam submetidos, assinaram um termo de consentimento livre e esclarecido. O presente estudo foi aprovado pelo Comitê de Ética em Pesquisa da Universidade Federal do Rio Grande do Norte (protocolo $n^{\circ}$. 540/11).

Para atender as finalidades do estudo os participantes foram divididos em quatro grupos etários 40-49 (G1), 50-59 (G2), 60-69 (G3) e $\geq 70$ anos (G4). Um cálculo amostral realizado a posteriori, baseado nos resultados de estudos prévios ${ }^{11,13,15}$, considerando um poder de estudo de $80 \%$ e nível de significância de $5 \%$, revelou a necessidade de uma amostra de no mínimo 85 participantes (40-49 anos, $\mathrm{n}=21$; 50-59 anos, $\mathrm{n}=21$; 60-69 anos, $\mathrm{n}=18 ; \geq 70$ anos, $\mathrm{n}=25$ ). O n necessário foi atingido em todos os grupos etários, exceto no grupo $\geq 70$ anos cuja amostra foi composta por 24 participantes.

Todos os participantes selecionados participaram de duas sessões de avaliação. $\mathrm{Na}$ primeira visita, foram realizadas entrevistas para aquisição de informações pessoais e, na sequência, foram executadas medidas antropométricas de massa corporal e estatura. A massa corporal foi medida em uma balança digital (Soehnle ${ }^{\circledast}$, Backnang, BW, Alemanha) e a estatura utilizando um estadiômetro (Sanny ${ }^{\circledR}$, São Bernardo do Campo, SP, Brasil) seguindo recomendações da literatura ${ }^{17}$. O índice de massa corporal (IMC) foi estabelecido pela razão entre a massa corporal (kg) e o quadrado da estatura (m). Na segunda visita foram avaliadas a força muscular e a ACR. A força muscular foi determinada por meio do teste de força de preensão manual (FPM) utilizando um dinamômetro hidráulico (Jamar ${ }^{\circledR}$, Jackson, MI, EUA). A avaliação da FPM foi realizada com o participante em posição ortostática com ombros e cotovelos relaxados e antebraço em posição semi-pronada. O dinamômetro foi ajustado individualmente de acordo com o tamanho da mão dos participantes. Foram concedidas três tentativas alternadas, com intervalo de $30 \mathrm{~s}$ para a mão direita e esquerda. O maior valor em quilogramas $(\mathrm{kgF})$ foi utilizado para as análises. Somente a FPM da mão direita foi adotada para as análises devido à ausência de diferenças significantes entre a força da mão direita e esquerda $(P>0,05)$. A ACR foi estimada pela distância percorrida no teste de caminhada de seis minutos (DPTC6min) ${ }^{18,19}$. O teste 
foi realizado seguindo as recomendações da American Thoracic Society ${ }^{19}$.

Inicialmente, o teste de Shapiro Wilk foi utilizado para a análise da distribuição dos dados. Na sequência o teste de Levene foi empregado para análise da homogeneidade das variâncias. Com base nessas informações análise de variância (ANOVA one-way) foi utilizada para as comparações entre os grupos. Para identificação das diferenças encontradas entre os grupos, o teste post hoc de Tukey foi adotado. Análise de correlação parcial foi utilizada para avaliar a correlação entre DPTC6min em cada grupo, de acordo com o gênero, e as variáveis idade, FPM, massa corporal, estatura e IMC. Adicionalmente, utilizou-se análise de regressão múltipla. Os dados foram processados no programa estatístico SPSS 19.0. Para todas as análises foi adotado um nível de significância de 5\%.

\section{Resultados}

Na tabela 1 são apresentados os valores de FPM e DPTC6min nos diferentes grupos etários. O G4 apresentou menores valores de FPM quando comparado aos grupos G1 e G2 $(P<0,05)$. Na DPTC6min diferenças estatisticamente significantes $(P<0,05)$ foram encontradas entre o grupo $\mathrm{G} 4$ e os demais grupos, com os menores valores sendo encontrados no grupo G4 (tabela 1).

TABELA 1 - Características gerais da amostra.

\begin{tabular}{lccccc}
\hline \multirow{2}{*}{ Variáveis } & \multicolumn{5}{c}{ Grupos } \\
\cline { 2 - 6 } & $\mathrm{G} 1(\mathrm{n}=64)$ & $\mathrm{G} 2(\mathrm{n}=79)$ & $\mathrm{G} 3(\mathrm{n}=69)$ & $\mathrm{G} 4(\mathrm{n}=24)$ & Geral $(\mathrm{n}=236)$ \\
\hline Idade (anos) & $44,9 \pm 2,8$ & $54,4 \pm 2,9$ & $63,8 \pm 3,0$ & $74,5 \pm 3,7$ & $56,6 \pm 9,8$ \\
Massa corporal (kg) & $73,3 \pm 13,3^{*}$ & $71,0 \pm 11,2$ & $71,0 \pm 12,6$ & $67,2 \pm 9,7$ & $71,2 \pm 12,1$ \\
\hline Estatura (m) & $1,59 \pm 0,08$ & $1,58 \pm 0,07$ & $1,58 \pm 0,08$ & $1,56 \pm 0,08$ & $1,58 \pm 0,08$ \\
IMC (kg/m²) & $29,06 \pm 5,16$ & $28,48 \pm 4,03$ & $28,51 \pm 3,94$ & $27,72 \pm 4,78$ & $28,57 \pm 4,40$ \\
\hline FPM (kg) & $30,9 \pm 8,8^{*}$ & $30,8 \pm 9,8^{*}$ & $29,4 \pm 8,9$ & $25,5 \pm 8,6$ & $29,9 \pm 9,3$ \\
\hline DPTC6min (m) & $569,9 \pm 63,9^{* *}$ & $565,9 \pm 73,3^{* *}$ & $542,4 \pm 70,6^{*}$ & $492,8 \pm 83,9$ & $552,7 \pm 74,5$ \\
\hline
\end{tabular}

IMC=índice de massa corporal; FPM=força de preensão manual; DPTC6min=distância percorrida no teste de caminhada de 6 minutos; $\mathrm{G} 1=40-49$ anos; $\mathrm{G} 2=50-59$ anos; $\mathrm{G} 3=60-69$ anos; $\mathrm{G} 4=\geq 70$ anos; ${ }^{*} \mathrm{P}<0,05$ vs. $\mathrm{G} 4 \mathrm{e}{ }^{* *} \mathrm{P}<0,001$ vs. G4.

Na Tabela 2 são apresentados os resultados da correlação parcial controlada por gênero entre a DPTC6min e idade, FPM, massa corporal, estatura e IMC. Somente no G4 ( $\geq 70$ anos) foi revelada correlação moderada, positiva e significante entre a DPTC6min e a FPM $(\mathrm{r}=0,51 ; P<0,05)$. Correlações significantes $(P<0,05)$ de fraca a moderada magnitude foram identificadas entre DPTC6min e as variáveis idade (G3), massa corporal (G1 e G2), estatura (G3) e IMC (G1 e G2).

TABELA 2 - Coeficiente de correlação parcial (r) entre a distância percorrida no teste de caminhada de seis minutos, idade, força de preensão manual, massa corporal, estatura e IMC, controlado por gênero.

\begin{tabular}{lccccc}
\hline \multirow{2}{*}{ Variáveis } & \multicolumn{5}{c}{ Grupos } \\
\cline { 2 - 6 } & $\mathrm{G} 1(\mathrm{n}=64)$ & $\mathrm{G} 2(\mathrm{n}=79)$ & $\mathrm{G} 3(\mathrm{n}=69)$ & $\mathrm{G} 4(\mathrm{n}=24)$ & Geral $(\mathrm{n}=236)$ \\
\hline Idade & 0,14 & $-0,23$ & $-0,24$ & $-0,30$ & $-0,38^{* *}$ \\
\hline FPM & 0,16 & 0,11 & 0,23 & $0,51^{*}$ & $0,29^{* *}$ \\
\hline Massa corporal & $-0,41^{* *}$ & $-0,21^{*}$ & 0,02 & 0,27 & $-0,13^{*}$ \\
\hline Estatura & 0,14 & 0,08 & $0,31^{* *}$ & $-0,63$ & $0,20^{* *}$ \\
\hline IMC & $-0,46^{* *}$ & $-0,27^{*}$ & $-0,15$ & $-0,21$ & $-0,24^{* *}$ \\
\hline
\end{tabular}

$F P M=$ força de preensão manual; IMC=índice de massa corporal; $G 1=40-49$ anos; $G 2=50-59$ anos; $\mathrm{G} 3=60-69$ anos; $\mathrm{G} 4=\geq 70$ anos. ${ }^{*} \mathrm{P}<0,05$ e ${ }^{* *} \mathrm{P}<0,001$. 
Na tabela 3 são apresentados os resultados da análise de regressão múltipla. Uma vez que diferentes variáveis foram correlacionadas com a DPTC6min em cada grupo, utilizou-se diferentes modelos de regressão múltipla. $\mathrm{O}$ maior $\mathrm{R}^{2}$ ajustado foi encontrado no G4 com o modelo incluindo FPM e gênero sendo capaz de explicar $47,4 \%$ da variância na distância percorrida $(P<0,05)$.

TABELA 3 - Modelos de predição do desempenho no teste de caminhada de 6 minutos a partir da análise de regressão múltipla.

\begin{tabular}{lccccc}
\hline & $\mathrm{G} 1(\mathrm{n}=64)$ & $\mathrm{G} 2(\mathrm{n}=79)$ & $\mathrm{G} 3(\mathrm{n}=69)$ & $\mathrm{G} 4(\mathrm{n}=24)$ & $\mathrm{Geral}(\mathrm{n}=236)$ \\
\cline { 2 - 6 } & $\beta$ & $\mathrm{B}$ & $\beta$ & $\beta$ & $\beta$ \\
\hline Constante & $765,0^{* *}$ & $732,6^{* *}$ & 116,7 & $370,9^{* *}$ & $762,0^{* *}$ \\
\hline Gênero & $-71,0^{* *}$ & $-94,3^{* *}$ & $-50,4^{*}$ & $-32,9$ & $-55,6^{* *}$ \\
\hline Idade & - & - & - & - & $-2,24^{* *}$ \\
\hline Estatura & - & - & $2,92^{* *}$ & - & 13,11 \\
\hline IMC & $-4,93^{* *}$ & $-3,72^{*}$ & - & - & $-4,34^{* *}$ \\
\hline FPM & - & - & - & $2,75^{*}$ & $1,94^{* *}$ \\
\hline $\mathrm{R}^{2}$ ajustado (\%) & $38,1^{* *}$ & $45,8 \%^{* *}$ & $35,8 \%^{* *}$ & $47,4 \%^{* *}$ & $46,4 \%^{* *}$ \\
\hline
\end{tabular}

G1=40-49 anos; G2=50-59 anos; G3=60-69 anos; G4= 70 anos; IMC=índice de massa corporal; FPM=força de preensão manual. ${ }^{*} P<0,05$ e ${ }^{* *} \mathrm{P}<0,001$.

\section{Discussão}

O principal achado foi que a força muscular foi positivamente associada a ACR em indivíduos a partir de 70 anos, independente do gênero e de características antropométricas. Adicionalmente, quando combinados em um modelo de regressão múltipla, a FPM e o gênero explicaram 47,4\% da variância na distância percorrida no teste de caminhada de 6-min no grupo $\geq 70$ anos. A importância da melhoria da força muscular para o aumento da ACR em idosos, encontrada no presente estudo, foi revelada por uma revisão recente conduzida por Ozaki et al. ${ }^{20}$ que encontraram que o treinamento resistido melhora o $\mathrm{VO}_{2 \max }$ em adultos jovens (20-40 anos) e idosos (> 60 anos), especialmente se o nível de $\mathrm{VO}_{2 \max }$ inicial for menor que $40 \mathrm{ml} \cdot \mathrm{kg}^{-1} \cdot \mathrm{min}^{-1}$ e $25 \mathrm{ml} \cdot \mathrm{kg}^{-1} \cdot \mathrm{min}^{-1} \mathrm{em}$ adultos e idosos, respectivamente.

Uma possível explicação para a associação entre força muscular e ACR parece estar relacionada a economia de movimento ${ }^{11}$. Cadore et al. ${ }^{11}$ mostraram uma correlação negativa entre força muscular (dinâmica e isométrica) e ativação muscular durante um teste de esforço máximo. Portanto, quanto mais forte for o idoso, menor o percentual da força máxima será utilizado durante o exercício aeróbio. Ademais, resultados de estudos transversais, longitudinais e de revisão suportam o fato de que a força muscular é um importante determinante para a redução da ACR com o avançar da idade ${ }^{1,2,14,15}$.

Com base na análise de correlação entre a FPM e DPTC6min para a amostra total e em cada grupo etário (Tabela 2) foi possível verificar que a combinação entre participantes de diferentes faixas etárias tende a subestimar a contribuição da força para ACR em idades mais avançadas, bem como superestimar em grupos mais jovens. O fato da correlação entre FPM e DPTC6min ter sido significante apenas no grupo mais idoso, provavelmente, se deva ao fato de que as reduções na massa muscular, força ${ }^{21,22} \mathrm{eVO}_{2 \max }{ }^{1,2}$ ocorrem de forma mais acentuada a partir da sétima década de vida. O coeficiente de determinação encontrado na combinação entre FPM e gênero para a estimativa da ACR no grupo etário mais avançado (G4) em nosso estudo guarda uma certa relação com os achados de Maranhão Neto et al. ${ }^{13}$ que, ao desenvolverem uma equação preditiva para estimativa da ACR sem a 
utilização de exercício em homens idosos com doenças cardiovasculares e metabólicas, revelaram uma capacidade de explicação de $79 \%$ para o $\mathrm{VO}_{2 \text { pico }}$ (idade $=44 \%$, ACR auto reportada $=13 \%$, FPM relativa ao peso $=22 \%$ ).

Enright et al..$^{23}$ ao analisarem uma amostra de 2.281 idosos relataram que para cada $10 \mathrm{~kg}$ adicional de FPM os participantes aumentaram a distância percorrida na caminhada em $14 \mathrm{~m}$ no TC6min. Tal resultado foi confirmado neste estudo cujo aumento encontrado foi na ordem de $56 \mathrm{~m}$ para cada $10 \mathrm{~kg}$ adicional de FPM em participantes acima de 70 anos.

Apesar da correlação positiva encontrada no presente estudo entre a força muscular e ACR no grupo mais idoso, em grupos mais jovens foram encontrados resultados diferentes daqueles relatados em estudos anteriores ${ }^{11,12,15}$. Tais resultados podem ser explicados, pelo menos em parte, pelos diferentes métodos utilizados, tanto para a avaliação da força quanto da ACR, bem como pelas diferenças nas amostras investigadas. Enquanto o presente estudo adotou os testes de FPM e de caminhada de seis minutos para a força muscular e da ACR, outros estudos avaliaram a força isométrica de membros inferiores ou força dinâmica em testes de 1-RM e a ACR em teste incremental em cicloergômetro ou esteira. Além disso, as análises se limitaram a grupos etários e gêneros específicos, diferente do delineamento adotado no presente estudo.

Os resultados do nosso estudo indicaram que é possível que a correlação entre força muscular e ACR, sobretudo em indivíduos de 40 a 69 anos, seja dependente do gênero. Fatores demográficos, antropométricos e fisiológicos influenciam o desempenho na DPTC6 $\mathrm{min}^{24}$, uma vez que indivíduos menores e mulheres apresentam menor comprimento de passada, enquanto obesos e idosos normalmente possuem menor massa muscular e percorrem uma menor distância nesse teste $e^{25}$. Nesse sentido, gênero, IMC, massa corporal e estatura mostraram-se determinantes da ACR nesse grupo etário.

Vale destacar que não somente os sistemas periféricos, mas também o sistema nervoso central é afetado pelo envelhecimento, de modo que idosos apresentam perda de unidades motoras, atrofia de axônios, desmilienização e modificação da transmissão dos sinais elétricos na junção neuromuscular ${ }^{26}$. Além disso, idosos apresentam um padrão diferenciado de ativação de áreas cerebrais comparado a indivíduos joven $s^{27}$. Todos esses fatores contribuem para a queda do desempenho muscular, afetando negativamente tanto a força muscular quanto a ACR em virtude da função neural diminuída.

O presente estudo apresenta algumas limitações que não devem ser desprezadas. O delineamento experimental (estudo transversal) não permite o estabelecimento de uma relação de causa e efeito. O baixo número de indivíduos nos grupos etários pode ter limitado o poder de análise da regressão múltipla. Além disso, o teste de caminhada de seis minutos permite uma estimativa de forma indireta da ACR, embora seja uma medida confiável ${ }^{28}$ e que possua uma boa correlação com o $\mathrm{VO}_{2 \max }{ }^{18,19}$. Vale ressaltar que o TC6min é de fácil aplicabilidade, bastante tolerável por parte dos avaliados e, talvez seja o teste que melhor representa as atividades diárias quando comparado a outros testes de caminhada. O TC6min também é uma alternativa para indivíduos que não podem ser submetidos a testes de esforço máximo devido ao seu baixo condicionamento físico, o que é relativamente comum em idosos. Por outro lado, o teste de FPM proporciona uma medida confiávele ${ }^{29}$, simples e sensível da força muscular, e pode representar a força global devido a sua correlação com a força de outros grupos musculares ${ }^{30}$. Adicionalmente, no presente estudo não foi avaliada a massa magra e gordura corporal, o que poderia ter aumentado o poder de predição dos modelos estatísticos utilizados no presente estudo. 
Como aplicação prática, nossos resultados recomendam a prática do treinamento de força muscular em idosos, para a melhoria da força e ACR, independentemente do $\mathrm{VO}_{2 \max }$. Tal modelo de treinamento tende a melhorar a estabilidade dinâmica, reduzindo o risco de quedas e, consequentemente, de fraturas nessa população, especificamente. Por fim, os resultados deste estudo sugerem que a força muscular pode ser adotada como um importante determinante da ACR em indivíduos a partir de 70 anos, independente de gênero e características antropométricas.

\section{Contribuições dos autores:}

DGSM: Concepção e delineamento, Procedimentos técnicos, Aquisição dos dados, Preparação do manuscrito, Redação do manuscrito, Análise estatística, Interpretação dos dados. HME: Concepção e delineamento, Revisão Crítica, Contribuição científica e intelectual. PMDA: Procedimentos técnicos, Aquisição dos dados, Análise estatística, Preparação do manuscrito, Revisão Crítica. LFFJR: Procedimentos técnicos, Aquisição dos dados, Análise estatística, Preparação do manuscrito, Revisão Crítica. LINN: Preparação do manuscrito, Análise estatística, Revisão crítica. AIF: Procedimentos técnicos, Aquisição dos dados, Preparação do manuscrito. CHNC: Procedimentos técnicos, Aquisição dos dados, Revisão Crítica. ECC: Concepção e delineamento, Revisão Crítica, Contribuição científica e intelectual. ESC: Revisão Crítica, Interpretação dos dados, Contribuição científica e intelectual. PTVF: Revisão Crítica, Contribuição científica e intelectual. AHO: Concepção e Delineamento, Contribuição científica e intelectual, Revisão Crítica. Todos os autores aprovaram a versão final do artigo.

\section{Agradecimentos}

Agradecemos aos voluntários pela participação voluntária no estudo. Este trabalho foi parcialmente financiado pelo Ministério da Educação e Cultura (PROEXT 2010 MEC/SESu, Programa Natal Ativa) a AHO, Concelho de Desenvolvimento Científico e Tecnológico (CNPq) e Coordenação de Aperfeiçoamento de Pessoal de Nível Superior.

\section{Referências}

1. Hawkins SA, Wiswell RA. Rate and mechanism of maximal oxygen consumption decline with aging: implications for exercise training. Sport Med. 2003;33(12):877-88.

2. Hawkins S, Marcell T. A longitudinal assessment of change in VO2max and maximal heart rate in master athletes. Med Sci Sports Exerc. 2001;33(10):1744-50.

3. Dodds RM, Roberts HC, Cooper C, Sayer AA. The Epidemiology of Sarcopenia. J Clin Densitom. 2015;18(4):461-6.

4. Janssen I, Heymsfield SB, Wang ZM, Ross R. Skeletal muscle mass and distribution in 468 men and women aged 18-88 yr. J Appl Physiol. 2000;89(1):81-8.

5. Syddall H, Cooper C, Martin F, Briggs R, Sayer AA. Is grip strength a useful single marker of frailty? Age Ageing. 2003;32(6):650-6.

6. Shephard RJ. Maximal oxygen intake and independence in old age. Br J Sport Med. 2009;43:342-6.

7. Blair SN, Cheng Y, Holder JS. Is physical activity or physical fitness more important in defining health benefits? Med Sci Sport Exerc. 2001;33(6):379-99.

8. Gale CR, Martyn CN, Cooper C, Sayer AA. Grip strength, body composition, and mortality. Int J Epidemiol. 2007;36(1):228-35.

9. Lee D-C, Sui X, Ortega FB, Kim Y-S, Church TS, Winett RA, et al. Comparisons of leisuretime physical activity and cardiorespiratory fitness as predictors of all-cause mortality in men and women. Br J Sport Med. 2011;45:504-10.

10. Volaklis KA, Halle M, Meisinger C. Muscular strength as a strong predictor of mortality: a narrative review. Eur J Intern Med. European Federation of Internal Medicine.; 2015;26(5):303-10. 
11. Cadore EL, Pinto RS, Alberton CL, Pinto SS, Lhullier FLR, Tartaruga MP, et al. Neuromuscular economy, strength, and endurance in healthy elderly men. J Strength Cond Res. 2011;25(4):997-1003.

12. Brentano MA, Cadore EL, Silva EM, Ambrosini AB, Coertjens M, Petkowics R, et al. Physiological adaptations to strength and circuit training in postmenopausal women with bone loss. J Strength Cond Res. 2008;22(6):1816-25.

13. Maranhão Neto GA, de Leon AP, Lira VA, Farinatti PT V. Assessment of cardiorespiratory fitness without exercise in elderly men with chronic cardiovascular and metabolic diseases. J Aging Res. 2012;2012:1-6.

14. Rosen MJ, Sorkin JD, Goldberg AP, Hagberg JM, Katzel LI. Predictors of age-associated decline in maximal aerobic capacity: a comparison of four statistical models. J Appl Physiol. 1998;84(6):2163-70.

15. Carvalho LP, Di Thommazo-Luporini L, Leheudre MA, Bonjorno JC, De Oliveira CR, Luporini RL, et al. Prediction of cardiorespiratory fitness by the six-minute step test and its association with muscle strength and power in sedentary obese and lean young women: a cross-sectional study. PLoS One. 2015;10(12):1-16.

16. Yanek LR, Vaidya D, Kral BG, Dobrosielski DA, Moy TF, Stewart KJ, et al. Lean mass and fat mass as contributors to physical fitness in an overweight and obese african american population. Ethn Dis. 2015;25(2):214-9.

17. Gordon CCC, Chumlea WCC, Roche AFF. Stature, recumbent length, and weight. In: Lohman TG, Roche AF, Martorell R, editors. Anthropometric standardization reference manual. Champaign: Human Kinetics Books; 1988. p. 3-8.

18. Burr JF, Bredin SSD, Faktor MD, Warburton DER. The 6-minute walk test as a predictor of objectively measured aerobic fitness in healthy working-aged adults. Phys Sportsmed. 2011;39(2):133-9.

19. American Thoracic Society. ATS statement: guidelines for the six-minute walk test. Am J Respir Crit Care Med. 2002; 166(1):111-7.

20. Ozaki H, Loenneke JP, Thiebaud RS, Abe T. Resistance training induced increase in VO2max in young and older subjects. Eur Rev Aging Phys Act. 2013;10(2):107-16.

21. Cooper C, Dere W, Evans W, Kanis JA, Rizzoli R, Sayer AA, et al. Frailty and sarcopenia: definitions and outcome parameters. Osteoporos Int. 2012;23(7):1839-48.

22. Fleg JL, Lakatta EG. Role of muscle loss in the age-associated reduction in VO2 max. J Appl Physiol. 1988;65(3):1147-51.

23. Enright PL, McBurnie MA, Bittner V, Tracy RP, McNamara R, Arnold A, et al. The 6-min walk test: a quick measure of functional status in elderly adults. Chest. 2003;123(2):387-98.

24. Britto RR, Probst VS, Dornelas De Andrade AF, Samora GAR, Hernandes NA, Marinho PEM, et al. Reference equations for the six-minute walk distance based on a Brazilian multicenter study. Brazilian J Phys Ther. 2013;17(6):556-63.

25. Dourado VZ. Reference equations for the 6-minute walk test in healthy individuals. Arq Bras Cardiol. 2011;96:128-38.

26. Manini TM, Hong SL, Clark BC. Aging and muscle: a neuron's perspective. Curr Opin Clin Nutr Metab Care. 2013;16(1):21-6.

27. Lin PP-Y, Lin S-IS, Chen J-JJJJ. Functional near infrared spectroscopy study of age-related difference in cortical activation patterns during cycling with speed feedback. IEEE Trans neural Syst Rehabil Eng. 2012;20(1):78-84.

28. Curb JD, Ceria-Ulep CD, Rodriguez BL, Grove J, Guralnik J, Willcox BJ, et al. Performancebased measures of physical function for high-function populations. J Am Geriatr Soc. 2006;54(5):737-42.

29. Hogrel J-Y. Grip strength measured by high precision dynamometry in healthy subjects from 5 to 80 years. BMC Musculoskelet Disord. BMC Musculoskeletal Disorders; 2015;16(139):1-11.

30. Wind AE, Takken T, Helders PJM, Engelbert RHH. Is grip strength a predictor for total muscle strength in healthy children, adolescents, and young adults? Eur J Pediatr. 2010;169(3):281-7.

ENDEREÇO PARA

CORRESPONDÊNCIA

ALEXANDRE HIDEKI OKANO

emaildookano@gmail.com
Departamento de Educação Física, Universidade Federal do Rio Grande do Norte, Campus Universitário BR 101 Lagoa Nova, CEP 59072-970. Natal, Rio Grande do Norte, Brasil.

Fone: +55 (84) 9813-4662 